\title{
ACESSIBILIDADE NA ATENÇÃO PRIMÁRIA: COMO AVALIAM OS PROFISSIONAIS DE SAÚDE?
}

\section{ACCESSIBILITY IN PRIMARY CARE: HOW DO HEALTH PROFESSIONALS EVALUATEIT?}

\section{ACCESIBILIDAD EN LA ATENCIÓN PRIMARIA: ¿CÓMO EVALUAN LOS PROFESIONALES DE LA SALUD?}

\author{
Jhayne Fonda Barra ${ }^{1}$, Mariana Coelho Moura Garcia ${ }^{2}$, Érika Andrade e Silva ${ }^{3}$, Isabel Cristina Gonçalves Leite ${ }^{4}$, Fernanda Moura
} Lanza $^{5}$, Angélica da Conceição Oliveira Coelho ${ }^{6}$

\begin{abstract}
RESUMO
Objetivo: avaliar a presença e extensão do atributo acesso de primeiro contato - componente acessibilidade, na perspectiva dos profissionais de atenção primária à saúde. Método: estudo transversal realizado em 62 unidades de atenção primária à saúde, por meio da autoaplicação do Primary Care Assessment Tool com 546 profissionais (60,3\% da população elegível). 0 banco de dados foi criado no software Epi-Info (versão 7), e a digitação realizada por entrada dupla. A análise foi realizada no software Statistical Package for the Social Sciences (versão 22). Para as comparações entre os grupos, foi utilizado o teste $U$ de Mann Whitney para amostras independentes. Resultados: a acessibilidade mostrou-se insatisfatória (média 3,5). Não foi identificada diferença significativa entre os modelos de atenção $(p=0,275)$. A área rural (média 3,9) apresentou melhor desempenho quando comparada à área urbana (média 3,5). Considerações finais: evidenciou-se necessidade de implementar estratégias relacionadas ao aspecto estrutural do serviço que busquem ampliação ao acesso.
\end{abstract}

Descritores: Atenção Primária à Saúde; Pesquisa sobre Serviços de Saúde; Acesso aos Serviços de Saúde.

\section{ABSTRACT}

Objective: to evaluate the presence and extent of the first contact access attribute - accessibility component, from the perspective of primary health care professionals. Method: cross-sectional study carried out in 62 primary health care units, through the selfapplication of the Primary Care Assessment Tool with 546 professionals (60.3\% of the eligible population). The database was created using Epi-Info software (version 7) and typing was performed by double entry. Analysis performed in the Statistical Package for the Social Sciences software (version 22). The Mann Whitney $U$ test for independent samples was used for comparisons between groups. Results: accessibility was unsatisfactory (average 3.5). No significant difference was identified between the models of care $(p=0.275)$. The rural area (average 3.9) performed better when compared with the urban area (average 3.5). Final considerations: there was a need to implement strategies related to the structural aspect of the service that seek to expand access. Descriptors: Primary Health Care; Health Services Research; Health Services Accessibility.

\section{RESUMEN}

Objetivo: evaluar la presencia y el alcance del atributo de acceso del primer contacto: componente de accesibilidad, desde la perspectiva de los profesionales de atención primaria de salud. Método: estudio transversal realizado en 62 unidades de atención primaria de salud, mediante la autoaplicación de la Herramienta de Evaluación de Atención Primaria con 546 profesionales (60,3\% de la población elegible). La base de datos se creó con el software Epi-Info (versión 7) y la escritura se realizó por doble entrada. Análisis realizado en el Paquete Estadístico para el software de Ciencias Sociales (versión 22). Se usó la prueba U de Mann Whitney para muestras independientes para las comparaciones entre grupos. Resultados: la accesibilidad fue insatisfactoria (promedio 3.5). No se identificaron diferencias significativas entre los modelos de atención $(p=0.275)$. El área rural (promedio 3.9) se desempeñó mejor en comparación con el área urbana (promedio 3.5). Consideraciones finales: era necesario implementar estrategias relacionadas con el aspecto estructural del servicio que buscan expandir el acceso.

Descriptores: Atención Primaria de Salud; Investigación sobre Servicios de Salud; Accesibilidad a los Servicios de Salud.

\footnotetext{
${ }^{1}$ Mestre em Enfermagem pela Faculdade de Enfermagem da Universidade Federal de Juiz de Fora. ${ }^{2}$ Enfermeira pela Universidade Federal de Juiz de Fora. ${ }^{3}$ Doutora em Saúde Coletiva pela Faculdade de Medicina da Universidade Federal de Juiz de Fora. Docente da Universidade Federal de Juiz de Fora. ${ }^{4}$ Doutora em Saúde Pública pela ENSP/FIOCRUZ. Docente da Universidade Federal de Juiz de Fora. ${ }^{5}$ Doutora em Enfermagem pela Escola de Enfermagem da Universidade Federal de Minas Gerais. Docente da Universidade Federal de São João del-Rei. ${ }^{6}$ Doutora em Enfermagem pela Escola de Enfermagem da Universidade Federal de Minas Gerais. Docente da Universidade Federal de Juiz de Fora.
}

Como citar este artigo:

Barra, JF, Garcia MCM , Silva , EA, et al . Acessibilidade na atenção primária: como avaliam os profissionais de saúde?

Revista de Enfermagem do Centro Oeste Mineiro. 2020;10:e3630. [Access__]; Available in: http//doi.org/10.19175/recom.v10i0.3630 


\section{INTRODUÇÃO}

Os serviços que compõem o Sistema Único de Saúde (SUS) encontram-se articulados e integrados à Rede de Atenção à Saúde (RAS), que tem as unidades de Atenção Primária à Saúde (APS) como coordenadoras e ordenadoras dos cuidados, as quais assumem o papel de primeiro contato preferencial dos usuários com 0 sistema ${ }^{(1)}$.

Dentre as formas de organização da APS reconhecidas pela Política Nacional de Atenção Básica (PNAB), a Estratégia Saúde da Família (ESF) é considerada prioritária para a sua consolidação no país ${ }^{(2)}$ Caracteriza-se por uma atenção focada nas demandas de saúde às famílias e comunidades, considerando o contexto sociocultural como fator que influencia a saúde e o bem-estar dos indivíduos ${ }^{(3-4)}$.

O fortalecimento dos sistemas de saúde pela expansão da APS vem sendo reconhecido, internacionalmente, como o meio mais efetivo para a melhoria das condições de saúde da população $0^{(5)}$. Por isso, a mensuração do desempenho dos serviços é uma das dez prioridades de pesquisa para a $\mathrm{APS}^{(6)}$. No contexto brasileiro, que ainda possui serviços de APS, na modalidade tradicional, há uma política, para consolidar esse ponto da RAS, por meio da ESF $^{(2)}$ O processo de avaliação de desempenho da APS destaca-se como importante ferramenta capaz de identificar as potencialidades $e$ as fragilidades desse serviço ${ }^{(7-8)}$.

$O$ enfoque tradicionalmente utilizado, para a avaliação da APS no Brasil e consoante com o arcabouço teórico da APS mundial ${ }^{(3)}$ e da PNAB $^{(2)}$, baseia-se na mensuração da presença e extensão dos seus atributos ${ }^{(7)}$ que são definidos como essenciais - acesso de primeiro contato, longitudinalidade, integralidade e coordenação e derivados - centralização na família, orientação para a comunidade e competência cultural ${ }^{(3)}$.

No entanto, apesar dos notórios avanços no acesso aos serviços de saúde, em decorrência da expansão da $\operatorname{ESF}^{(9)}$, os estudos avaliativos brasileiros, realizados com profissionais da APS, apontam o acesso como atributo que apresenta maior dificuldade de operacionalização e consolidação ${ }^{(4,10-12)}$.

Esse atributo envolve dois componentes: a acessibilidade e a utilização. A dimensão acessibilidade é um componente estrutural do serviço e está condicionada à disponibilidade do serviço perante as necessidades dos usuários, incluindo a localização próxima à comunidade, os horários e dias de funcionamento e a flexibilidade aos atendimentos de demanda espontânea das unidades. Já a dimensão utilização - componente processual - está relacionada à percepção dos usuários de que o serviço é acessivel e considerado referência ${ }^{(3)}$.

Considerando que, para a realização das ações de saúde, os serviços precisam implementar, no cotidiano de trabalho, os elementos estruturantes da $\operatorname{APS}^{(3)}$ e que há necessidade de fortalecimento da APS vigente, no que se refere à prática de seus atributos essenciais $^{(1-2) .} O$ presente estudo tem como objetivo: avaliar a presença e extensão do atributo acesso de primeiro contato componente acessibilidade, na perspectiva dos profissionais da APS.

\section{MÉTODO}

Trata-se de um estudo transversal de caráter avaliativo, aprovado pelo Comitê de Ética em Pesquisa, CAAE 33363314.9.0000.5147, parecer número 3.383.322, atendendo às determinações da Resolução 466/12 e 510/2016 do Conselho Nacional de Saúde, que estabelece as diretrizes e normas regulamentadoras de pesquisas envolvendo seres humanos.

O cenário de pesquisa foi constituído por 62 das 63 unidades de APS do município de Juiz de Fora, sendo 47 urbanas e 15 rurais. Foram convidados a participar da pesquisa todos os profissionais médicos, enfermeiros, técnicos de enfermagem e agentes comunitários de saúde (ACS) que atuavam nas equipes de Saúde da Família (eSF) e equipes de Atenção Básica (eAB). Foram excluídos do estudo os profissionais que estavam de férias regulamentares, licença médica ou afastados; os que se recusaram a participar do estudo e aqueles que não foram encontrados após três tentativas.

A coleta de dados ocorreu entre março de 2018 e fevereiro de 2019, mediante autoaplicação do instrumento Primary Care Assessment Tool (PCATool), versão profissionais, validado para o contexto brasileiro ${ }^{(8)}$ e um questionário elaborado, para fins desta pesquisa, que coletou informações sobre o perfil sociodemográfico e qualificação dos profissionais.

Os participantes foram informados dos objetivos da pesquisa, procedimentos, riscos e benefícios, por meio do Termo de Consentimento Livre e Esclarecido (TCLE). Aos profissionais, que 
não tiveram interesse em participar, foi-lhes instruído apenas devolver o questionário e o TCLE em branco. Todos os profissionais de uma unidade de APS se recusaram a participar do estudo (motivos não foram explicitados à pesquisadora principal), o que justifica a realização da pesquisa em 62 unidades de APS.

O PCATool contém 77 itens divididos em oito componentes. $\mathrm{Na}$ versão profissionais, o PCATool avalia apenas o componente acessibilidade do atributo acesso, o qual é constituído por nove itens (A1, A2, A3, A4, A5, A6, A7, A8 e A9). As respostas possíveis, para cada item, são baseadas na escala de likert: "com certeza $\operatorname{sim}^{\prime}$ (valor=4); "provavelmente sim" (valor=3); "provavelmente não" (valor=2); "com certeza não" (valor=1) e "não sei/ não lembro" $(\text { valor }=9)^{(8)}$.

Procedeu-se à inversão de valores do item A9, visto que foi formulado a fim de que quanto maior o valor atribuído menor é a orientação para a APS. O escore do atributo foi calculado pela média aritmética simples dos valores das respostas para cada pergunta e, posteriormente, transformado em escala contínua de 0 a 10, utilizando a fórmula [(escore obtido - 1) X 10] / 3. Para a análise do resultado do escore do atributo foi utilizada a mesma metodologia do manual do PCAtool, que determina que valores iguais ou maiores que 6,6 representam alta orientação para a $\mathrm{APS}^{(8)}$.A variável dependente foi o escore do atributo acessibilidade, e as variáveis independentes foram compostas pelo sexo, idade, profissão, qualificação e experiência profissional.
O banco de dados foi criado no software Epi-Info (versão 7) e a digitação realizada por entrada dupla. Em seguida, foi avaliada a consistência dos dados pela função data compare, com o intuito de eliminar falhas de digitação. A tabulação dos dados foi realizada no software Microsoft Excel (2013), e a análise estatística no software Statistical Package for the Social Sciences for Windows (SPSS) - versão 22.

As variáveis qualitativas foram descritas como frequências absolutas e relativas, e as variáveis quantitativas, conforme método de análise dos dados recomendado pelo PCATool ${ }^{(8)}$,como média e desvio-padrão.

Para a estatística analítica, testou-se a normalidade da variável quantitativa por meio do teste de Kolmogorov-Sminorv. Descartada a normalidade dos dados, utilizou-se o teste não paramétrico $U$ de Mann Whitney para a comparação dos escores entre os grupos. A todos os testes, foi adotado um nível de significância de $p<0,05$.

\section{RESULTADOS E DISCUSSÃO}

Participaram do estudo 546 profissionais, alocados em 62 unidades de APS (47 urbanas e 15 rurais). Ressalta-se que todos os profissionais de uma unidade de APS se recusaram a participar do estudo (motivos não foram explicitados à pesquisadora principal). Foram excluídos 27 profissionais que estavam de férias regulamentares, 10 por licença médica ou afastamento e 321 que se recusaram a participar do estudo e/ou não foram encontrados após três tentativas. O perfil dos participantes do estudo encontra-se na Tabela 1.

Tabela 1 - Perfil dos participantes estratificado por modalidade de serviço, Juiz de Fora, 2019.

\begin{tabular}{|c|c|c|c|c|}
\hline \multirow{2}{*}{ Variáveis Qualitativas } & \multicolumn{2}{|c|}{$e^{*}{ }^{*}(n=57)$} & \multicolumn{2}{|c|}{$\mathrm{eSF}^{\dagger}(n=489)$} \\
\hline & № & $\%$ & № & $\%$ \\
\hline \multicolumn{5}{|l|}{ Sexo } \\
\hline Feminino & 47 & 82 & 431 & 88 \\
\hline Masculino & 9 & 16 & 47 & 10 \\
\hline Não informado & 1 & 2 & 11 & 2 \\
\hline \multicolumn{5}{|l|}{ Profissional } \\
\hline Médico & 11 & 19 & 41 & 8 \\
\hline Enfermeiro & 16 & 28 & 74 & 15 \\
\hline Técnico de enfermagem & 30 & 53 & 63 & 13 \\
\hline ACS & & & 311 & 64 \\
\hline \multicolumn{5}{|l|}{ Treinamento $^{\S}$ para atuar APS $^{\ddagger}$} \\
\hline Sim & 26 & 46 & 321 & 66 \\
\hline Não & 20 & 35 & 64 & 13 \\
\hline Não informado & 11 & 19 & 104 & 21 \\
\hline \multicolumn{5}{|l|}{ Vínculo trabalhista em outro serviço } \\
\hline Sim & 14 & 25 & 32 & 7 \\
\hline Não & 29 & 51 & 354 & 72 \\
\hline Não informado & 14 & 25 & 103 & 21 \\
\hline
\end{tabular}

Fonte: Dados da pesquisa (2019).

Notas:*equipe de Atenção Básica. ${ }^{\dagger}$ equipe de Saúde da Família. ${ }^{\ddagger}$ Atenção Primária à Saúde. ${ }^{\S}$ Capacitação dos profissionais para atuarem na APS pela Secretaria Municipal de Saúde. 
Com relação à qualificação dos profissionais, identificou-se que nove $(9,7 \%)$ técnicos de enfermagem e $45(14,5 \%)$ ACS possuem curso superior; 27 (51,9\%) médicos e 49 $(54,4 \%)$ enfermeiros possuem especialização em saúde da família; sete $(13,5 \%)$ médicos e três $(3,3 \%)$ enfermeiros $(7,0 \%)$ possuem residência em saúde da família.

A média de idade dos profissionais foi de 47,54 anos (desvio-padrão $\pm 10,2$ ). A maior idade foi 70 anos e a menor 25 anos.
A análise dos itens que constitui o atributo acessibilidade indicou baixa orientação do serviço para o atributo na perspectiva dos profissionais (média 3,5; desvio-padrão $\pm 1,1$ ), assim como em outros estudos que utilizaram o mesmo instrumento ${ }^{(4,10-11-12-13-14)}$. A descrição de cada item do componente acessibilidade encontra-se na Tabela 2.

Tabela 2 - Avaliação da presença e extensão do atributo acesso - componente acessibilidade - e seus respectivos itens na perspectiva dos profissionais da APS, Juiz de Fora, MG, Brasil, 2019.

\begin{tabular}{|c|c|c|c|}
\hline Acessibilidade & $\begin{array}{c}\text { Escore } \\
\text { Médio/Desvio- } \\
\text { padrão }\end{array}$ & IC $95 \%$ & $\begin{array}{l}\text { \% Alto } \\
\text { Escore } \\
(>6,6)\end{array}$ \\
\hline A1- O serviço está aberto sábado ou domingo & $0,6( \pm 1,5)$ & $0,5-0,7$ & 2,1 \\
\hline A2- Está aberto pelo menos alguns dias até as 20 horas & $1,2( \pm 2,7)$ & $0,9-1,4$ & 9,4 \\
\hline $\begin{array}{l}\text { A3- Se o Serviço de Saúde está aberto e o usuário fica doente, alguém o atende } \\
\text { no mesmo dia }\end{array}$ & $8,0( \pm 2,3)$ & $7,8-8,2$ & 93,6 \\
\hline $\begin{array}{l}\text { A4- Os pacientes conseguem aconselhamento rápido pelo telefone quando o } \\
\text { Serviço de Saúde está aberto }\end{array}$ & $6,8( \pm 3,0)$ & $6,6-7,1$ & 79,9 \\
\hline $\begin{array}{l}\text { A5- Existe algum número de telefone, para o qual os pacientes possam ligar, } \\
\text { quando o Serviço de Saúde está fechado }\end{array}$ & $2,2( \pm 3,4)$ & $1,9-2,5$ & 19,2 \\
\hline $\begin{array}{l}\text { A6- Quando o Serviço de Saúde está fechado, aos finais de semana, e o paciente } \\
\text { fica doente alguém do Serviço de Saúde o atende no mesmo dia }\end{array}$ & $0,8( \pm 1,9)$ & $0,6-0,9$ & 4,4 \\
\hline $\begin{array}{l}\text { A7- Quando o Serviço de Saúde está fechado à noite, e o paciente fica doente } \\
\text { alguém do Serviço de Saúde o atende naquela noite }\end{array}$ & $0,6( \pm 1,7)$ & $0,5-0,8$ & 3,5 \\
\hline A8- É fácil, para os pacientes, marcar uma consulta de revisão de saúde & $7,6( \pm 2,5)$ & $7,4-7,8$ & 90,6 \\
\hline $\begin{array}{l}\text { A9- Em média, os pacientes têm que esperar mais de } 30 \text { minutos para serem } \\
\text { atendidos pelo médico ou enfermeiro }\end{array}$ & $3,8( \pm 2,7)$ & $3,6-4,1$ & 26,9 \\
\hline
\end{tabular}

Fonte: Dados da pesquisa (2019).

A acessibilidade é característica essencial da APS e responsável por permitir a entrada do usuário no sistema de saúde, garantindo um atendimento a suas necessidades e à sua família. Associa-se a fatores como: disponibilidade de atendimento, localização do estabelecimento e aos entraves de comunicação entre equipes e usuários ${ }^{(3)}$.

Uma revisão da literatura, realizada por autores que validaram o PCATool, para o contexto brasileiro, sugere a necessidade de atualização do item acessibilidade do instrumento, incluindo ou adaptando itens, como exemplo, novos meios de comunicação entre profissional e usuário, como e-mail e aplicativos $^{(15)}$. Ao analisar o instrumento original proposto por Starfield, para analisar o serviço de APS americano ${ }^{(16)}$, percebe-se que os autores brasileiros realizaram a adaptação fiel ao instrumento, sem efetuar uma adaptação cultural das características organizacionais da APS brasileira.
Nesse sentido, o instrumento validado ao Brasil $^{(8)}$ avalia características dos serviços de APS que não são realidades, no extenso território do país, como atendimentos aos sábados e domingos; horário de funcionamento até as 20 horas; número de telefone para o qual os usuários podem ligar quando o serviço está fechado. Tais características foram responsáveis pela baixa qualificação do atributo, uma vez que não são contemplados na realidade do município em estudo.

É importante salientar que, habitualmente, as pessoas trabalham nos horários de atendimento convencionais das unidades, o que torna comum que outros serviços da RAS - como as unidades de pronto atendimento (UPA), acabem por funcionar como porta de entrada, em decorrência de inflexibilidade na programação das unidades ${ }^{(12,14)}$. Ademais, a dinâmica das equipes de APS, de forma geral, prioriza atendimentos de rotina, e a comunicação dos usuários com o serviço geralmente requer a 
presença física ${ }^{(4)}$. O serviço somente é considerado acessível, quando a população percebe a conveniência dos aspectos que envolvem os dias e horários de atendimento e a tolerância para as atividades não programadas ${ }^{(3)}$.

A PNAB recomenda que o horário de funcionamento das unidades facilite o acesso da população ao serviço, situação avaliada nos itens A1, A2, A6 e A7. A referida portaria propõe carga horária mínima de 40 horas semanais (no mínimo cinco dias da semana) e permite a pactuação de horários alternativos mediante demandas da população. A responsabilidade da análise das demandas territoriais cabe à gestão municipal. Cada profissional de eSF deve cumprir obrigatoriamente a carga horária mínima supracitada. Já as $\mathrm{e} A B$ têm maior flexibilidade: carga horária mínima por profissional de dez horas e mínima por categoria de 40 horas. Dessa forma, as eAB podem ser compostas por até três profissionais de cada categoria ${ }^{(2)}$.

Percebe-se que a proposta da PNAB permite vários arranjos às $\mathrm{A} A \mathrm{~B}$. A flexibilização $d a$ jornada de trabalho dos profissionais põe em risco a continuidade do cuidado, o estabelecimento de vínculo entre usuários e equipe $e$ fortalece $o$ modelo curativo individual $^{(17)}$. Portanto, quando em seu texto dispõe que: "os horários e dias de funcionamento devem ser organizados de modo que garantam amplamente o acesso, o vínculo entre as pessoas e profissionais, a continuidade, coordenação e longitudinalidade do cuidado"(2, p. 14), em termos, contradiz-se.

Outro fato que merece destaque, pois pode comprometer intimamente o acesso da população à APS, é a indefinição no quantitativo de ACS por equipe, pois é um trabalhador do território, que conhece a dinâmica da comunidade e, em consequência, é um facilitador da aproximação entre o serviço e a comunidade ${ }^{(17)}$.

Apesar desses entraves e com intuito de ampliar essa oferta aos serviços primários, algumas iniciativas pontuais vêm sendo desenvolvidas no país. Em Recife, um modelo denominado "Upinhas 24 horas" vem sendo implementado desde o ano de 2013 para reduzir as disparidades do acesso às eSF. Como diferencial, esse serviço oferece horário de funcionamento estendido a consultas e situações de urgência nas unidades de APS. O modelo garante, na maioria das vezes, um atendimento pelo profissional de saúde, mas esbarra na questão da continuidade do cuidado, pois é pouco provável que, após o horário convencional, o usuário seja atendido pela sua equipe ${ }^{(18)}$.

Pela Portaria GM n. 930, de 15 de maio de 2019, o Ministério da Saúde instituiu o "Programa Saúde na Hora", com o intuito de ampliar a cobertura das eSF e os atendimentos, além de reduzir os atendimentos de baixo risco nos hospitais e unidades de pronto atendimento. A proposta é que a carga horária semanal seja de, no mínimo, 60 horas, incluindo cinco horas aos sábados ou domingos. Aplica-se aos profissionais médicos, enfermeiros e cirurgiões dentistas e, ainda, prevê incentivo financeiro por parte do governo federal às unidades que aderirem ao programa ${ }^{(19)}$.

Como o atual secretário de Atenção Primária à Saúde do MS é o pesquisador principal dos estudos de validação do PCATool, para o contexto brasileiro, pode-se inferir que o Programa Saúde na Hora foi elaborado, mediante os resultados das pesquisas brasileiras que utilizaram o PCATool, já que todas mostraram baixa orientação do atributo acesso, indicando fragilidades no período de funcionamento da APS.

Outra importante inciativa a fim de fomentar o acesso, é a implantação do acolhimento nas unidades, preconizada como diretriz da Política Nacional de Humanização. Sua institucionalização ocorre pela escuta qualificada de demandas não programadas dos usuários com o objetivo de garantir o acesso adequado a cada necessidade ${ }^{(20)}$.

O tempo que os usuários esperam, para receber atendimento, foi outro fator que contribuiu para a baixa qualificação do atributo. Percebe-se que a facilidade e a agilidade do acesso ao serviço, ainda, são desafios organizacionais a serem superados ${ }^{(21)}$.

Em Juiz de Fora, não há flexibilidade, nos dias e horários de atendimento das unidades, que pontualmente funcionam, durante a semana, de segunda a sexta-feira, das sete às 11 horas e de 13 às 17 horas, com exceção das quintas-feiras, em que o serviço funciona até as 15 horas, para reunião de equipe. Aos finais de semana e no período noturno, o acesso da população aos serviços públicos de saúde fica condicionado aos hospitais e Unidades de Pronto Atendimento (UPA), que funcionam 24 horas. A comunicação 
da população com o serviço fica restrita a esse arranjo organizacional.

O fluxograma de atendimento das unidades, basicamente, acontece da seguinte forma: usuários com demanda programada chegam ao início do expediente e aguardam o atendimento; a demanda espontânea é encaminhada para o acolhimento; e os procedimentos e demais atendimentos são realizados por ordem de chegada, respeitando as prioridades. Essa organização do serviço pode estar associada ao elevado tempo de espera para o atendimento, principalmente, no que condiz com o manejo da demanda programada, o que poderia ser minimizado com agendamento de consultas por horário.

Uma revisão integrativa analisou 42 estudos que utilizaram o PCATool entre 2001 e 2016 e apresentou o comportamento de cada atributo da APS no contexto nacional e internacional. Desses estudos, 40,5\% foram realizados no Brasil. Ressalta-se que apenas na Columbia - Carolina do Sul, Seul - Coreia do Sul, Changsha - China e na Região da Catalunha Espanha, a acessibilidade alcançou forte orientação para a APS ${ }^{(15)}$, portanto verifica-se que a operacionalização do atributo ainda é incipiente no Brasil e no mundo.

Com relação à comparação do desempenho do atributo acessibilidade entre os modelos de atenção, o presente estudo não apontou diferença estatisticamente significativa $(p=0,275)$. As eAB apresentaram escore médio de 3,6 (desvio-padrão $\pm 1,0$ ) e às eSF escore médio de 3,5(desvio-padrão $\pm 1,1$ ). Vale destacar que as eAB apresentaram escore significativamente maior com relação ao item $A 4$, conforme a Tabela 3.

Tabela 3 - Comparação do atributo acesso - componente acessibilidade - e seus respectivos itens, na perspectiva dos profissionais, entre as eSF e eAB, Juiz de Fora, MG, Brasil, 2019.

\begin{tabular}{|c|c|c|c|}
\hline \multirow{2}{*}{ Acessibilidade } & \multicolumn{2}{|c|}{ Escore Médio (Desvio-Padrão) } & \multirow{2}{*}{ Valor de $p^{\ddagger}$} \\
\hline & eSF $^{*}(n=489)$ & $e^{A B^{\dagger}}(n=57)$ & \\
\hline A1- O serviço está aberto sábado ou domingo & $0,6( \pm 1,6)$ & $0,5( \pm 1,2)$ & 0,926 \\
\hline A2- Está aberto pelo menos alguns dias até as 20 horas & $1,2( \pm 2,8)$ & $0,7( \pm 1,9)$ & 0,280 \\
\hline $\begin{array}{l}\text { A3- Se o Serviço de Saúde está aberto e o usuário fica doente, alguém o } \\
\text { atende no mesmo dia }\end{array}$ & $8,0( \pm 2,3)$ & $7,9( \pm 2,2)$ & 0,526 \\
\hline $\begin{array}{l}\text { A4- Os pacientes conseguem aconselhamento rápido pelo telefone } \\
\text { quando o Serviço de Saúde está aberto }\end{array}$ & $6,7( \pm 3,0)$ & $7,9( \pm 2,5)$ & 0,003 \\
\hline $\begin{array}{l}\text { A5- Existe algum número de telefone para o qual os pacientes podem ligar } \\
\text { quando o Serviço de Saúde está fechado }\end{array}$ & $2,0( \pm 3,4)$ & $1,9( \pm 3,6)$ & 0,255 \\
\hline $\begin{array}{l}\text { A6- Quando o Serviço de Saúde está fechado, aos finais de semana, e o } \\
\text { paciente fica doente, alguém do Serviço de Saúde o atende no mesmo dia }\end{array}$ & $0,8( \pm 2,0)$ & $0,8( \pm 1,7)$ & 0,442 \\
\hline $\begin{array}{l}\text { A7- Quando o Serviço de Saúde está fechado à noite, e o paciente fica } \\
\text { doente, alguém do Serviço de Saúde o atende naquela noite }\end{array}$ & $0,6( \pm 1,8)$ & $0,6( \pm 1,6)$ & 0,773 \\
\hline A8- É fácil para os pacientes marcar uma consulta de revisão de saúde & $7,6( \pm 2,6)$ & $8,0( \pm 2,4)$ & 0,205 \\
\hline $\begin{array}{l}\text { A9- Em média, os pacientes têm que esperar mais de } 30 \text { minutos para } \\
\text { serem atendidos pelo médico ou enfermeiro }\end{array}$ & $3,8( \pm 2,7)$ & $4,4( \pm 3,1)$ & 0,145 \\
\hline
\end{tabular}

Fonte: Dados da Pesquisa (2019).

Notas:*equipe de Atenção Básica. ' equipe de Saúde da Família. ${ }^{\ddagger}$ Teste $U$ de Mann-Whitney de amostras independentes.

Um estudo comparativo entre os modelos de atenção, realizado em Piracicaba, no estado de São Paulo, apontou melhor desempenho da acessibilidade nas $\mathrm{eAB}$, sinalizando que 0 acesso não obteve melhoras com a implantação das $\mathrm{eSF}^{(13)}$, corroborando com o resultado deste estudo.

Destaca-se que, no munícipio em questão, ambos os modelos de atenção possuem a mesma gestão e, basicamente, são organizados pelos mesmos princípios, fato que talvez tenha colaborado com o resultado.

Contudo, apesar de denotadas essas dificuldades, em ambos os modelos, estudos indicam a saúde da família como estratégia capaz de produzir melhores resultados nos serviços primários ${ }^{(14,22)}$. Entretanto não condiz com o texto da nova PNAB, que expressa a "desconstrução de um compromisso com a expansão da saúde da família e do sistema público"(17, p.14), pois, ao mesmo tempo, em que considera a estratégia como prioritária, para consolidação da APS no país, reconhece e incentiva outras modalidades de organização(2), colocando em risco o SUS e seus princípios ${ }^{(17)}$.

Além disso, a PNAB não prevê valorização diferenciada entre as equipes ${ }^{(17)}$, 0 que compromete a transição das eAB para eSF, no território brasileiro, como preconizado pela política. É fato que a existência simultânea de 
ambos os modelos de APS é um empecilho para a reversão do modelo assistencial curativo e individual $^{(4)}$.

O resultado deste estudo reforça que, apesar da expansão do acesso, em decorrência da expansão das eSF, é evidente a necessidade de avanços e superação das barreiras que impedem sua concretização, sejam elas geográficas ou organizacionais $^{(11)}$.
A acessibilidade mostrou-se maior na área rural (escore médio de 3,9; e desvio-padrão $\pm 1,2$ ) que na área urbana (escore médio de 3,5 e desvio-padrão $\pm 1,1)$, não apresentando diferença significativa $(p=0,073)$. A descrição dos escores de cada item do componente acessibilidade e suas comparações encontra-se na Tabela 4.

Tabela 4 - Comparação do atributo acesso - componente acessibilidade - e seus respectivos itens, na perspectiva dos profissionais, entre as áreas urbana e rural, Juiz de Fora, MG, Brasil,2019

\begin{tabular}{|c|c|c|c|}
\hline \multirow[t]{2}{*}{ Acessibilidade } & \multicolumn{2}{|c|}{ Média (DP) } & \multirow[t]{2}{*}{ Valor de $p^{*}$} \\
\hline & Urbana $(n=507)$ & Rural (n=39) & \\
\hline A1- O serviço está aberto sábado ou domingo & $0,6( \pm 1,6)$ & $0,3( \pm 1,0)$ & 0,332 \\
\hline A2- Está aberto pelo menos alguns dias até as 20 horas & $1,2( \pm 2,8)$ & $0,6( \pm 2,0)$ & 0,173 \\
\hline $\begin{array}{l}\text { A3- Se o Serviço de Saúde está aberto e o usuário fica doente, alguém o } \\
\text { atende no mesmo dia }\end{array}$ & $8,0( \pm 2,3)$ & $8,6( \pm 1,7)$ & 0,119 \\
\hline $\begin{array}{l}\text { A4- Os pacientes conseguem aconselhamento rápido pelo telefone } \\
\text { quando o Serviço de Saúde está aberto }\end{array}$ & $6,7( \pm 3,0)$ & $8,6( \pm 2,2)$ & $<0,001$ \\
\hline $\begin{array}{l}\text { A5- Existe algum número de telefone para o qual os pacientes podem } \\
\text { ligar quando o Serviço de Saúde está fechado }\end{array}$ & $2,1( \pm 3,3)$ & $2,4( \pm 4,1)$ & 0,786 \\
\hline $\begin{array}{l}\text { A6- Quando o Serviço de Saúde está fechado, aos finais de semana, e o } \\
\text { paciente fica doente, alguém do Serviço de Saúde o atende no mesmo } \\
\text { dia }\end{array}$ & $0,8( \pm 1,1)$ & $0,9( \pm 1,1)$ & 0,761 \\
\hline $\begin{array}{l}\text { A7- Quando o Serviço de Saúde está fechado à noite, e o paciente fica } \\
\text { doente, alguém do Serviço de Saúde o atende naquela noite }\end{array}$ & $0,6( \pm 1,8)$ & $0,8( \pm 1,9)$ & 0,735 \\
\hline A8- É fácil para os pacientes marcar uma consulta de revisão de saúde & $7,6( \pm 2,6)$ & $8,9( \pm 2,1)$ & $<0,001$ \\
\hline $\begin{array}{l}\text { A9- Em média, os pacientes têm que esperar mais de } 30 \text { minutos para } \\
\text { serem atendidos pelo médico ou enfermeiro }\end{array}$ & $3,9( \pm 2,7)$ & $3,9( \pm 3,2)$ & 0,909 \\
\hline
\end{tabular}

Fonte: Dados da Pesquisa (2019).

Notas: *Teste U de Mann-Whitney de amostras independentes.

Estudo realizado em Diamantina, Minas Gerais, com profissionais e usuários, apontou avaliação significativamente maior para o atributo na área rural em comparação à área urbana ${ }^{(23)}$.

Considerando que os usuários da região rural enfrentam constantemente dificuldades de acesso (a distância entre a população e a fonte de atenção, meios de transporte escassos, rotatividade e escassez de profissionais), sem considerar, na maioria das vezes, que são áreas extremamente vulneráveis ${ }^{(24)}$, acredita-se que, neste estudo, a qualificação dos profissionais tenha se associado positivamente ao resultado, visto que $70 \%(n=24)$ dos profissionais que atuam nessas equipes receberam treinamento, para atuar na APS, e todos os profissionais de nível superior possuem especialização em saúde da família.

Destaca-se que a formação voltada para a Saúde da Família está entre os fatores que mais se associam ao bom desempenho da APS e deve ser estimulada e valorizada ${ }^{(25)}$, portanto modelos de atenção voltados para os contextos das comunidades, considerando suas práticas e saberes tradicionais, são indispensáveis ao atendimento das necessidades de saúde ${ }^{(3)}$.

Além disso, tanto a oferta quanto a procura pelo serviço primário podem se dar em diferentes circunstâncias. Na área urbana, a população conta com maior variedade de serviços, em contrapartida, na área rural, a APS pode ser a única fonte de atenção àquela comunidade. A própria dispersão geográfica reduz a procura por outros serviços como fonte regular de atenção ${ }^{(23)}$.

Os serviços de APS rural e urbano do município, embora organizados pelos mesmos princípios e gestão, diferem-se quanto á adscrição da população. As unidades rurais são responsáveis por um quantitativo menor de usuários, por isso, acredita-se que a facilidade em receber aconselhamento pelo telefone e para marcar consulta, tenham apresentado significância nessa área.

Como limitação do estudo, espera-se que a auto aplicação pode ter colaborado com o elevado quantitativo de recusas, visto que os resultados mostrados são de $28,1 \%$ dos médicos elegíveis 
para o estudo; $69,2 \%$ dos enfermeiros; $58,9 \%$ dos técnicos de enfermagem e $72,1 \%$ dos ACS. No entanto optou-se por esta metodologia, buscando garantir a cobertura do estudo, em todas as unidades, considerando os recursos físicos e financeiros disponíveis e o tempo hábil para a sua execução.

Como contribuições para o avanço do conhecimento, o estudo demonstrou que a avaliação é um instrumento capaz de direcionar e embasar a tomada de decisão, em busca de maior efetividade da atenção, ao apontar as características do serviço que dificultam a acessibilidade.

O Brasil é um país em desenvolvimento, com uma APS não consolidada em sua totalidade, mas vem buscando constantemente superar os desafios e avançar na concretização dos princípios e diretrizes do SUS ${ }^{(4)}$.

\section{CONSIDERAÇÕES FINAIS}

A pesquisa mostrou que, segundo a experiência dos profissionais médicos, enfermeiros, técnicos de enfermagem e ACS, o componente acessibilidade do atributo acesso de primeiro contato está presente, nos serviços de APS do município de Juiz de Fora, porém com um desempenho insatisfatório (escore inferior a 6,6). Ambos os modelos de atenção apresentaram baixa orientação para o atributo. A área rural apresentou maior orientação, quando comparada à área urbana, no entanto não se identificou diferença estatisticamente significante.

Apenas três itens apresentaram alta orientação para a APS (escores acima de 6,6), sendo o que se refere ao atendimento, no mesmo dia, quando o usuário fica doente durante o horário de funcionamento do serviço de saúde (item A3); à possibilidade de aconselhamento rápido por telefone quando a unidade está aberta (item A4) e à facilidade de marcação de consulta para a revisão de saúde (item $A 8$ ).

Considerando que o item acessibilidade do PCATool não considera a realidade dos atendimentos da APS de Juiz de Fora e que isso tenha colaborado à baixa orientação desses serviços, acredita-se que simplesmente buscar novas adaptações e validações não seja a melhor estratégia para avaliar o desempenho do atributo acesso. As fragilidades medidas no PCATool merecem ser discutidas e avaliadas pelos gestores locais com o intuito de traçar estratégias que reduzam essas disparidades e deem visibilidade à APS como porta de entrada.

Sugere-se adequar os dias e horários de funcionamento dos serviços às necessidades da comunidade. Além disso, vislumbra-se a adesão ao Programa Saúde na Hora como um importante passo em busca dessa ampliação.

Enfatiza-se, também, a importância de investimentos na capacitação e qualificação de todos profissionais que integram as equipes de APS.

Sugerem-se futuros estudos comparativos com a percepção dos usuários do serviço, visto que o objetivo primordial da assistência à saúde é a melhoria nas suas condições de vida e saúde.

Conclui-se que o estudo possibilitou identificar a presença e extensão da acessibilidade no serviço de APS local, na perspectiva dos profissionais, apontando contribuições para o aprimoramento do serviço. Esses apontamentos têm influência, na melhoria das condições de saúde da comunidade e na redução das disparidades de acesso e utilização do serviço, bem como auxiliam a tomada de decisão e elaboração de intervenções com essa finalidade.

\section{REFERÊNCIAS}

1- Brasil. Ministério da Saúde. Portaria no 4.279, de 30 de dezembro de 2010. Estabelece diretrizes para a organização da Rede de Atenção à Saúde no âmbito do Sistema Único de Saúde (SUS). Diário Oficial da União 2010.

2- Brasil. Ministério da Saúde. Departamento de Atenção Básica. Portaria MS/GM no 2436, de 21 de setembro de 2017. Aprova a Política Nacional de Atenção Básica, estabelecendo a revisão de diretrizes para a organização da Atenção Básica, no âmbito do Sistema Único de Saúde (SUS). Diário Oficial da União 2017.

3- Starfield B. Atenção primária: Equilíbrio entre necessidades de saúde, serviços e tecnologia. Brasília: UNESCO; 2002.

4- Silva SA, Baitelo TC, Fracolli LA.Avaliação da Atenção Primária à Saúde: $A$ visão de usuários e profissionais sobre a Estratégia de Saúde da Família. RevLatinoamEnferm.2015;23(5):979-87. DOI: 10.1590/0104-1169.0489.2639

5- World Health Organization (WHO). Declaration of Astana. Global Conference on Primary Health Care. Astana: World Health Organization; 2018. 
6- O'Neil B, Aversa V, Rouleau K, Lazare K, Sullivan F, Persaud N. Identifying top 10 primary care research priorities from international stakeholders using a modified Delphi method. PLoSOne 2018;13(10):e0206096. DOI: 10.1371/journal.pone.0206096

7- Fracolli LA, Gomes MFP, Nabão FRZ, Santos MS, Cappellini VK, Almeida ACC. Instrumentos de avaliação da Atenção Primária à Saúde: Revisão de literatura e metassíntese. Ciênc Saúde Colet. 2014;19(12):4851-60.DOI:

10.1590/1413812320141912.00572014

8- Brasil. Ministério da Saúde. Secretaria de Atenção em Saúde. Manual do instrumento de avaliação da atenção primária à saúde: Primarycare assessment tool PCATOOL. Brasília: Ministério da Saúde; 2010.

9- Viacava F, Oliveira RAD, Carvalho CC,Laguardia J,Bellido JG. SUS: Oferta, acesso e utilização de serviços de saúde nos últimos 30 anos.Ciênc. Saúde Colet. 2018;23(6):1751-62. DOI: 10.1590/141381232018236.06022018

10- Vieira NF, Rodrigues RN, Niitsuma ENA, Lanza FM, Lana FCF. Avaliação da Atenção Primária: Comparativo entre o desempenho global e as ações de hanseníase. RevEnfermCent-Oeste Min. 2019;9:e2896. DOI: 10.19175/recom.v9i0.2896

11- Batista VCL, Ribeiro LCC, Ribeiro CDAL, Paula FA, Araújo A. Avaliação dos atributos da atenção primária à saúde segundo os profissionais de saúde da família. Sanare2016 [citado em 20 jul2019]; 15(2):87-93. Availibe in: https://sanare.emnuvens.com.br/sanare/article/vi ew/1042

12- Santos NA, Lima DR, Gontijo MKB, Martins MA, Leite GR, Silva LA. Avaliação dos atributos da atenção primária por profissionais de saúde. Rev APS 2017;20(3):339-348. DOI: 10.34019/18098363.2017.v20.15964

13- Cesar MC, Campos GWS, Montebelo MIL, Sarmento G. Avaliação da atenção primária no município de Piracicaba, SP, Brasil. Saúde debate 2014;38(nesp):296-306. DOI: 10.5935/01031104.2014S022

14- Ferreira VD, Oliveira JM, Maia MAC, Santos JS, Andrade RD, Machado GAB. Avaliação dos atributos da Atenção Primária à Saúde em um Município Mineiro. Esc Anna Nery RevEnferm. 2016;20(4): e20160104.DOI: h10.5935/14148145.20160104

15- D'Avila OP, Pinto LFS, Hauser L, Gonçalves MR, Harzheim E. O uso do PrimaryCare Assessment Tool (PCAT): Uma revisão integrativa e proposta de
atualização.Ciênc Saúde Colet. 2017;22(3):855-65. DOI: 10.1590/1413-81232017223.03312016

16- Cassady CE, Starfield B, Hurtado MP, Berk RA, Nanda JP, Friedenberg LA. Measuring consumer experiences with Primary Care. Pediatrics 2000 [citado em 20 jul 2019]; 105(4Pt2):9981003.Availibe in: https://pediatrics.aappublications.org/content/105 LSupplement 3/998.short

17- Morosini MVGC, Fonseca AF, Lima LD. Política Nacional de Atenção Básica 2017: Retrocessos e riscos para o Sistema Único de Saúde. Saúde Debate 2018;42(116):11-24. DOI:10.1590/01031104201811601

18- Pessoa BHS, Gouveia EAH, Correia IB. Funcionamento 24 horas para Unidades de Saúde da Família: Uma solução para ampliação de acesso? Um ensaio sobre as "Upinhas" do Recife. RevBrasMedFamComunidade 2017;12(39):1-9. DOI: $10.5712 / \mathrm{rbmfc} 12$ (39)1529

19- Brasil. Ministério da Saúde. Portaria $n^{\circ}$ 930, de 15 de maio de 2019. Institui o Programa Saúde na Hora, que dispõe sobre o horário estendido de funcionamento das Unidades de Saúde da Família, altera a Portaria no 2.436/GM/MS, de 2017, a Portaria de Consolidação no 2/GM/MS, de 2017, a Portaria de Consolidação no 6/GM/MS, de 2017, e dá outras providências. Diário Oficial da União2019.

20- Martins ACT, Paula AP, Cardoso JR, Borges MIG, Botelho MB. O Projeto AcolheSUS na Atenção Primária à Saúde do Distrito Federal, Brasil. Ciênc Saúde Colet. 2018;24(6):2095-2103. DOI:10.1590/1413-81232018246.08492019

21. Oliveira VBCA, Verissímo MLOR. Assistência à Assistência à saúde da criança segundo suas famílias: Comparação entre modelos de Atenção Primária. RevEscEnferm USP 2015;49(1):30-6. DOI: 10.1590/S0080-623420150000100004

22- Araújo PJ, Vieira CS, Oliveira BRG, Gaiva MA, Rodrigues RM. Avaliação dos atributos essenciais da Atenção Primária à Saúde da criança. RevBrasEnferm. 2018;71(3):1447-54. DOI: 10.1590/0034-7167-2017-0569

23- Silva GS, Alves CRL. Avaliação do grau de implantação dos atributos da atenção primária à saúde como indicador da qualidade da assistência prestada às crianças. CadSaúde Pública 2019;35(2):e00095418. DOI: 10.1590/0102311x00095418

24- Pessoa VM, Almeida MM, Carneiro FF. Como garantir o direito à saúde para as populações do campo, da floresta e das águas no Brasil? Saúde 
Debate 2018;42(nesp):302-14.DOI: 10.1590/0103$11042018 \mathrm{~s} 120$

25- Turci MA, Lima-Costa MF, Macinko J. Influência de fatores estruturais e organizacionais no desempenho da atenção primária à saúde em Belo Horizonte, Minas Gerais, Brasil, na avaliação de gestores e enfermeiros. Cad. SaúdePública 2015;31(9)1941-52. DOI: 10.1590/0102-311X00132114

Nota: Artigo proveniente da Dissertação de Mestrado intitulada "Avaliação dos Atributos da Atenção Primária à Saúde na perspectiva dos profissionais", do Programa de PósGraduação Stricto Sensu em Enfermagem da Universidade Federal de Juiz de Fora.

Recebido em: 13/12/2019

Aprovado em: 08/04/2020

Endereço para correspondência:

Angélica da Conceição Oliveira Coelho

Faculdade de Enfermagem. Rua José Lourenço Kelmer, s/n Campus Universitário Bairro São Pedro - CEP: 36036-900 - Juiz de Fora-MG

E-mail: angelica.fabri@ufjf.edu.br 